\title{
A Surface Phase Transition of Supported Gold Nanoparticles
}

\author{
Anton Plech, ${ }^{*}$ Roland Cerna, Vassilios Kotaidis, Florian Hudert, \\ Albrecht Bartels, and Thomas Dekorsy
}

Department of Physics and Center for Applied Photonics, University of Konstanz, Universitätsstrasse 10, D-78457 Konstanz, Germany

\begin{abstract}
A thermal phase transition has been resolved in gold nanoparticles supported on a surface. By use of asynchronous optical sampling with coupled femtosecond oscillators, the Lamb vibrational modes could be resolved as a function of annealing temperature. At a temperature of $104^{\circ} \mathrm{C}$ the damping rate and phase changes abruptly, indicating a structural transition in the particle, which is explained as the onset of surface melting.
\end{abstract}

Metallic nanoparticles are in the focus of fundamental and applied research. They show an optical response in the visible region that depends strongly on size, shape, and local dielectric environment, ${ }^{1}$ making them appealing for nanoscale sensor applications. The interaction with light shows interesting peculiarities, such as ultrafast relaxation dynamics, coupling to nonradiative processes, or near field enhancement. ${ }^{2}$ In particular nanoscale gold particles have received strong attention due to their versatile synthesis protocols, ${ }^{3}$ shape transformations, or binding to biological molecules. ${ }^{4}$ They served also to understand the generic structural properties that scale with size or shape. The melting point depression with decreasing particle size represents one of the prominent features, arising from the change in ratio between surface and bulk atoms and the reduction in binding energy. Since the seminal publication of Buffat and Borel ${ }^{5}$ several improvements of the description have been made, while the general relation of the melting point depression $\Delta T_{\mathrm{mp}} \propto 1 / D$ as a function of size $D$ remains valid. One of these points is the existence of surface melting, i.e., a coexistence of a liquid-like layer of atoms at the surface with the solid core material at temperatures below the respective melting point. .,7 $^{6}$

While this effect seems to be very basic, there is still a lack of unambiguous experimental proof regarding the details, in particular for gold nanoparticles.

Inasawa et al. ${ }^{8}$ have tried to investigate the shape transformation of elongated particles on a surface induced by static heating (ex situ) and concluded that a transformation into spheres takes places at temperatures as low as $400{ }^{\circ} \mathrm{C}$. A similar, very recent study has shown that nanorods with a large aspect ratio of the axes can be transformed at 200

\footnotetext{
* Corresponding author. E-mail: anton.plech@uni-konstanz.de.
}

${ }^{\circ} \mathrm{C}$ into spheres and partial relaxation occurs much earlier $\left(100{ }^{\circ} \mathrm{C}\right) .{ }^{9}$ Surface melting is taken as the origin for these relaxations.

It should also be borne in mind that the relaxation of rods could also be caused by other forces, as has been shown by laser-induced melting, ${ }^{10}$ where the onset correlates with dislocations in the interior, as the rods are subjected to a large uniaxial strain due to surface tension.

Hartland et al. have also reported on several experiments, which aimed at the structural transformations on a very short time scale, initiated by femtosecond excitation. ${ }^{11,12}$ The vibrational response of the particles has been recorded by optical pump-probe techniques, reflecting the mechanical properties of the particles. While the change of the elastic constants of the spheres with temperature seems to be as expected, no abrupt changes could be detected owing to the melting transition of the particles. This has been attributed to the interference with surface melting or to saturable absorption. In contrast, time-resolved X-ray scattering gave proof for the melting transition, ${ }^{13}$ while also revealing competitive channels for structural relaxations, such as ablation. ${ }^{14}$ From a theoretical point of view the phase diagram has been calculated by a mean field approach by Sakai. ${ }^{6}$ The melting line $T_{\mathrm{mp}}(D)$ as a function of particle size $D$ is intercepted by the surface melting line, both of which meet at a critical particle size, below which surface melting is suppressed. The large particle limit should end at the bulk values of melting and surface melting. This behavior is correlated with experiments on lead particles, ${ }^{15}$ where surface melting starts around $70 \%$ of $T_{\mathrm{mp}}$. For gold, however, it is known that surface melting is extremely dependent on the crystal orientation. ${ }^{16}$ While the (110) surface premelts considerably below the melting point, the (111) surface does 
not show surface melting. The behavior of the (100) surface is more special, showing reconstruction below the melting point. ${ }^{17} \mathrm{~A}$ very low premelting temperature (38\% of the bulk $T_{\mathrm{mp}}$ ) has been reported in transmission electron microscopy experiments of platinum nanoparticles. ${ }^{18}$

We show in the present investigation, that there exists a structural transition at very low temperature (30\% of $T_{\mathrm{mp}}$ ) using pump-probe spectroscopy to follow the vibrational motion of supported particles by heating the supporting substrate. The transition is in principle reversible, while at larger temperatures sintering of the particles adds an irreversible component.

As has been shown by several groups in the past years, metal nanoparticles can be electronically excited with ultrafast laser pulses followed by electron-phonon coupling and lattice heating. ${ }^{19,20}$ As the electron-phonon coupling is considerably faster than the low-frequency eigenmodes of the particles, this heating can launch oscillatory motion of the particle shape. An analytical description or the frequencies of the eigenmodes for spheres is available, dating back to the early description of Lamb. ${ }^{21,22}$ They are sorted according to modal "quantum" numbers, describing radial, angular, and torsional motion. In optical spectroscopy only the first two are relevant in the detection scheme. ${ }^{23}$ Using integer numbers proportional to the number of nodes, which appear in the strain profile $u(\mathbf{r}, t)$ in radial and angular direction, classifies the oscillations: the ground mode with spherical symmetry as $(0,0)$ mode (breathing mode), the $(1,0)$ mode has a node at a spherical surface in the interior of the particles, and the $(0,2)$ mode has two nodal lines at the surface, following a oblate to prolate shape modulation.

The optical response of vibrationally excited particles follows the periodic change in electron density, which modifies the position of the plasmon resonance. ${ }^{24}$ The response also contains an ultrafast part, originating from the nonequilibrium heating of the electronic subsystem and decays within about 1 ps. ${ }^{19,25}$

We have employed a recently developed pump-probe technique based on two asynchronously locked femtosecond lasers. ${ }^{26,27}$ In high-speed asynchronous optical sampling (ASOPS) the time delay between pump and probe pulses is realized by an off-set frequency of the repetition rate of the two femtosecond lasers. Our system is based on two Kerrlens mode-locked Ti:sapphire femtosecond lasers with $1 \mathrm{GHz}$ repetition rate (Model GigaJet Twin, Gigaoptics GmbH, Germany) which are stabilized at a fixed repetition rate difference of $10 \mathrm{kHz}$ by an electronic stabilization circuit. In contrast to conventional femtosecond pump-probe methods ASOPS does not rely on delaying one pulse relative to the other by using a mechanical translation stage, hence circumventing problems due to spot size variation or pointing instabilities as usually present when using long mechanical delay lines. With ASOPS the inverse of the repetition rate of the pump-laser (1 ns) is sampled at $10 \mathrm{kHz}$ with a time resolution of $100 \mathrm{fss}^{27,28}$ Using a single fast detector (125 $\mathrm{MHz}$ bandwidth) for the reflected probe pulse in conjunction with a fast $\mathrm{A} / \mathrm{D}$ converter (100 MHz bandwidth), one can achieve a signal-to-noise ratio of $10^{7}$ in less than $1 \mathrm{~min}$

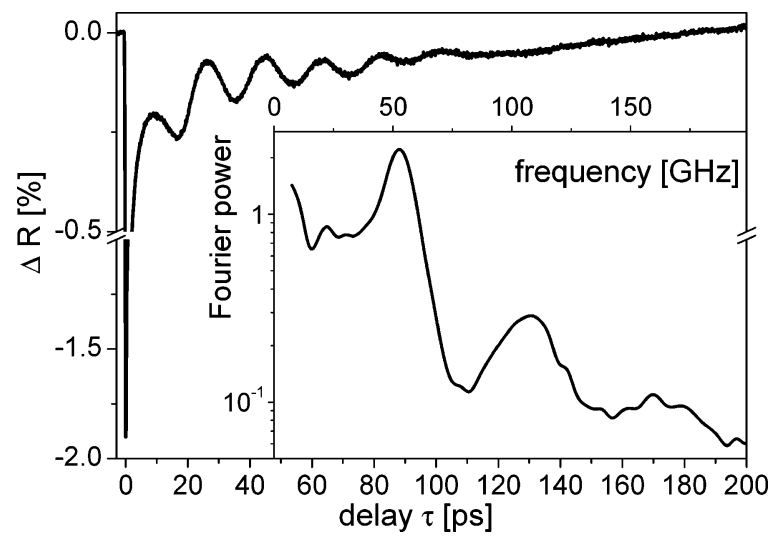

Figure 1. Transient reflectivity change as function of delay time between pump pulse and probe pulse. The inset shows the Fourier transform of the transient reflectivity trace with two clear peaks at 52 and $109 \mathrm{GHz}$, identified as $(0,0)$ and $(1,0)$ vibrational modes. The $(0,2)$ mode would be located at $21 \mathrm{Ghz}$, where a considerable Fourier intensity appears.

sampling time. No mechanical modulation (chopping) of pump or probe is necessary. Both lasers are independently tunable in their wavelength which simplifies blocking stray light of the pump pulse before the detector by a bandpass filter. In our experiments we used pump pulses of 150-320 $\mathrm{mW}$ average power (150-320 $\mathrm{pJ}$ energy per pulse only as compared to experiments performed with conventional femtosecond oscillators with $100 \mathrm{MHz}$ repetition rate and pulse energies in the $10 \mathrm{~nJ}$ range) at $820 \mathrm{~nm}$ and probe pulses of $27 \mathrm{~mW}$ at $796 \mathrm{~nm}$. Both pulses have pulse durations below 50 fs.

The samples consist of silicon wafers ((100) surface) which are covered by a submonolayer of gold particles. The particles are purchased from BBInternational (U.K.) with a nominal diameter of $61.5 \mathrm{~nm}$ and a size dispersion of about $8 \%$. The method of self-assembly by polyelectrolyte layers has been described elsewhere. ${ }^{29}$ It should be noted, that surface diffusion leads to aggregations of particles. These aggregations lead to an electronic coupling of the individual plasmon resonances ${ }^{1,30}$ and the appearance of a broad absorption band from the initial resonance ( $535 \mathrm{~nm}$ in water) to the infrared and peaking at $600-750 \mathrm{~nm}$. This effect enhances the sensitivity on the vibrations at the probe wavelength. Both beams are focused and overlapped onto the surface by a $50 \mathrm{~mm}$ lens, and the reflected light is detected by a photodiode after proper spatial and polarization filtering. Here we used s polarization for the pump and $\mathrm{p}$ polarization for the probe pulses, which however is not essential for the detection of the vibrational modulations.

At the same time the wafer is heated by a resistive heater and the temperature is ramped up at $1 \mathrm{~K} / \mathrm{min}$ by using a controller with Pt100 probe (Lakeshore). The ASOPS system allows to record a full transient within a minute, so the temperature can be regarded as constant. The transient reflectivity change for the probe light is shown in Figure 1. It shows an abrupt decrease within the first 100 fs of delay. This decrease recovers within $1.6 \mathrm{ps}$ to be followed by a number of smaller undulations. It can be interpreted analogously to previous studies of nanoparticle excitation. ${ }^{19,24}$ The 


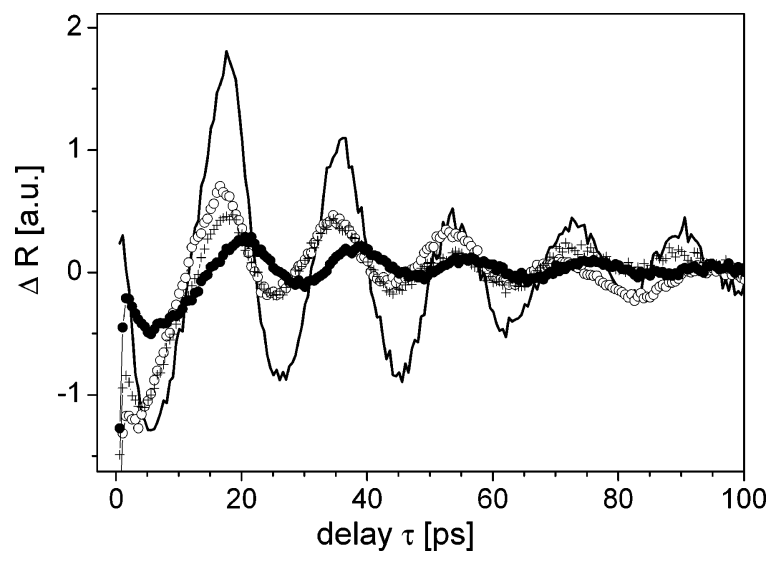

Figure 2. Oscillatory part of the transient reflectivity at different temperatures below $T_{\text {trans }}$ at $(-) 33^{\circ} \mathrm{C}$, (O) $65^{\circ} \mathrm{C}$, and $(+) 87^{\circ} \mathrm{C}$ and above the transition at $\left(-115^{\circ} \mathrm{C}\right.$ at $150 \mathrm{~mW}$ pump laser power.

extinction increases due to the heating of the conduction electrons and recovers partly due to the injection of the excess energy into the lattice. The particles start to oscillate around the new equilibrium size, which causes periodic changes of the extinction. In our case the reflectivity change follows the extinction with inverse sign. The presented method is not background free; therefore the long time decay, which shows the relaxation of the lattice, may be biased. We attribute the bias to the presence of additional diffuse scattering signal from the particles. From an inspection the Fourier spectrum of the oscillations, the $(0,0)$ and the $(1,0)$ modes can be identified, while the $(0,0)$ mode dominated the spectrum. Signatures of the $(0,2)$ modes are seen but not well-resolved in frequency above the background (inset of Figure 1). We will restrict ourselves to the discussion of the oscillatory part of the $(0,0)$ mode. which is extracted in Figure 2 It can be modeled by the function

$$
I(\tau) \propto \cos (2 \pi / T \tau-\phi) \exp \left(-\tau^{2} / \tau_{\mathrm{r}}^{2}\right)
$$

with the oscillation period $T=\pi D /\left(\eta c_{1}\right)$, the damping time $\tau_{\mathrm{r}}$, and a phase relation between the excitation and the oscillatory movement $\phi$. The present data show a period of $T=19 \mathrm{ps}$, which is exactly what would be expected for 62 $\mathrm{nm}$ sized particles, when using the eigenvalue solution for $\eta$ of the $(0,0)$ vibration ${ }^{21}$ according to

$$
\eta \cot \eta=1-\frac{\eta^{2}}{4\left(c_{\mathrm{t}} / c_{1}\right)^{2}}
$$

which depends on the velocities of longitudinal and transversal sound $c_{1}$ and $c_{\mathrm{t}}$, respectively. It has been shown, that the decay of the oscillation can be due to homogeneous damping (phonons decay within one particle) ${ }^{31}$ or due to an inhomogeneous damping of an ensemble of different sized particles. ${ }^{24}$ For a narrow size distribution the damping rate as written in eq 1 is obtained, with $\tau=50 \mathrm{ps}$ in good agreement with the value expected from the size dispersion of $8 \%$. Moreover the square in the exponential fits the data

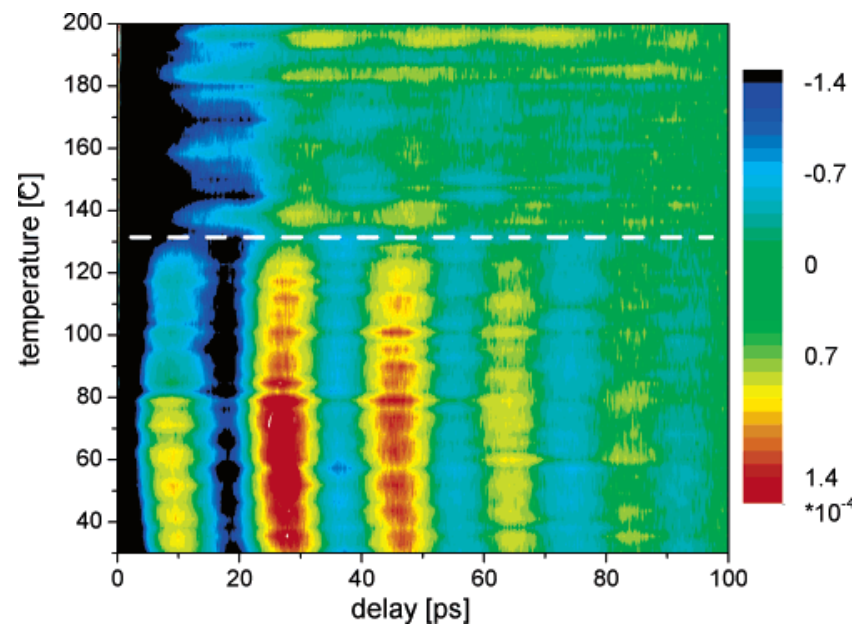

Figure 3. False color plot of the oscillatory part of the transient reflectivity as a function of delay and temperature at $308 \mathrm{~mW}$ pump laser power. At the dashed line a sudden change in the vibrational reaction sets in, including a phase shift and an increase in damping.

better than a linear exponent in $\tau$. Apparently the homogeneous damping is weaker than the observed rate.

When following the oscillations for increasing temperature (see surface plot of transient reflectivity in Figure 3), the frequency and the damping rate remain stable for a range in temperature, while the phase shows a small drift. The phase in general is related to the nature of the excitation. As it is found to be typically close to zero in other studies, the mechanism is generally classified as displacive. The rapid heating is thereby imposing a new equilibrium size immediately after excitation, around which the actual size oscillates. In detail, it is known that for metal nanoparticles there are two different mechanisms present, one from the direct pressure of the hot electron gas and one from the new equilibrium size due to thermal expansion. ${ }^{23,32,33}$ On the other hand, the phase may also depend on the boundary conditions, in particular the particles internal structure.

At a distinct temperature the phase of the oscillation changes abruptly from about -0.2 to 0.6 radians and then stabilizes. At the same time the damping rate increases by a factor of 2. The period is less affected but tends to increase slightly. This increase becomes more pronounced for higher temperatures, then finally the damping diverges, which reflects the vanishing of the oscillations.

We have repeated the experiment at different pump laser powers to elucidate the role of the continuous wave heating caused by the pump itself. There is indeed an influence of the pump power on the transition temperature, however, counterintuitively showing an increase of $T_{\text {trans }}$ from $104{ }^{\circ} \mathrm{C}$ at $150 \mathrm{~mW}$ to $135^{\circ} \mathrm{C}$ at $320 \mathrm{~mW}$. There is no straightforward explanation for this effect, as an increased laser power should also increase the substrate temperature, thereby lowering the apparent transition temperature as read by the temperature controller. We suspect, that the higher power exerts a force on the coupled particles, which then decouple and do not contribute to the signal.

The transition is also visible weakly in the continuous wave reflectivity of the surface, which has been recorded 


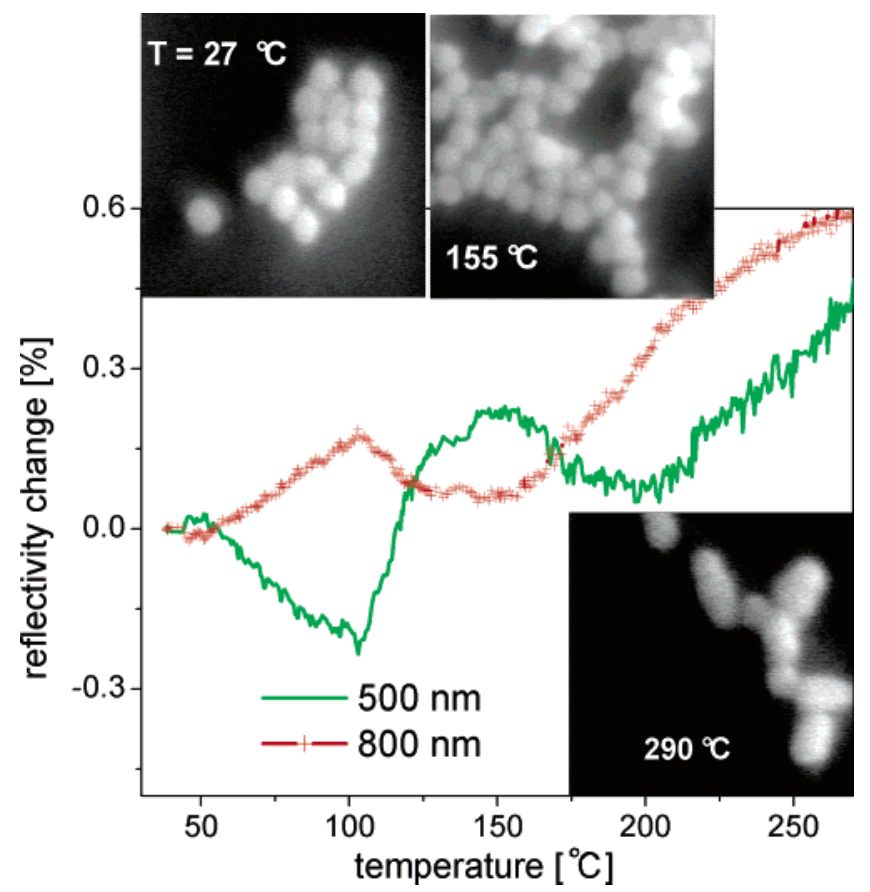

Figure 4. Continuous wave reflectivity change of s-polarized light at $500 \mathrm{~nm}$ (green line) and $800 \mathrm{~nm}$ (red line with crosses) as a function of the temperature. Insets: Scanning electron micrographs of the particle morphology after tempering for the indicated temperatures. Each micrograph shows a field of $590 \times 590 \mathrm{~nm}^{2}$.

by a halogen source and a fiber spectrometer (Ocean Optics). Figure 4 displays the traces at 500 and $800 \mathrm{~nm}$ as function of heater temperature. Both show a cusp at around $103{ }^{\circ} \mathrm{C}$. At $500 \mathrm{~nm}$ the plasmon resonance within a single particle is probed, which tends to decrease at lower temperature due to progressive coupling of particles, while it increases again, when particles start to sinter after surface melting. The opposite trend exists for $800 \mathrm{~nm}$, which probes a certain fraction of coupled particles ${ }^{34}$ according to their size. At higher temperatures, where sintering dominates both the reflectivities at 500 and $800 \mathrm{~nm}$ increase due to the larger particles with increased oscillator strength.

To account for this shift, the temperature has been rescaled to a reduced temperature $T_{\text {red }}=T / T_{\text {trans }}-1$ relative to the determined $T_{\text {trans }}$ in Figure 5. It can be seen that the different curves match nicely and also tiny features are reproduced. In addition a third experiment has been performed, where the temperature ramp has been stopped closely above the transition temperature and then reversed to cool down the sample. It shows that indeed the transition is to a large extent reversible both in the damping time and in the phase. Electron microscopy images at room temperature, right above $T_{\text {trans, }}$, and at the highest temperature $\left(290^{\circ} \mathrm{C}\right)$ help to understand this phenomenon. The apparent spherical shape and the assembly of particles are not strongly perturbed, when crossing the transition. In contrast at the highest temperature $\left(290{ }^{\circ} \mathrm{C}\right)$ the particles sinter together and lose their spherical shape completely.

We conclude that the transition is based on structural changes involving the restructuring of the surface. The sharp phase change when crossing the transition temperature can be explained by an abrupt occurrence of a surface melting layer. The particles develop a core-shell structure with an elastic core and a viscous shell. The resulting oscillation frequency will not be much affected, while slowing slightly down, if a very thin liquid shell is assumed. This will hold for a symmetric coupling of the extensional move in both core and shell. ${ }^{35}$ However the boundary condition changes abruptly due to the reduced elasticity of the shell. The damping feels the existence of a viscous shell as well and will increase. Hodak et al. ${ }^{36}$ have performed pump-probe experiments on gold core particles with a thin lead shell from 4 to 20 monolayers. These results show the modification of the frequency as calculated later by Sader et al. ${ }^{37}$ and (although not explicitly mentioned) a change of the phase upon lead addition on the surface. We extracted a phase change from -0.1 to 0.4 radians from Figure 2 in ref 36 for the 4 monolayer coverage. An analytic description of such coupled driven vibration with boundary condition is not available yet, and these results could stimulate further studies.

An alternative explanation could be that any (undetermined) contamination (possibly hydrocarbons) on the surface of the particles would be pyrolyzed upon heating, which in turn would change the size of the particles and their vibrational properties. Please note that the particles were initially not ligand protected. However, in this case one should expect a reduced damping rate and the vibrational frequency should increase due to the mass reduction, which is not in agreement with the observations. Finally the reversibility of the process cannot be explained by such a heterogeneous process.

The final sintering process at higher temperature reflects the increase in mobility and the morphology change, which is facilitated by the surface melting layer. Looking closer at the small features in damping and particular in the phase at $T<T_{\text {trans }}$, which are reproducible in the different runs, we cannot exclude additional subtle transformation taking place at the same time, such as surface reconstruction, etc.

The observed frequency change can give an estimate for the thickness of a surface melting layer. Sader et al. have given an expression for the frequency of core-shell particles based on the elastic properties of the material for two limiting cases. One case is valid in the limit of a thin shell, the other of a small core relative to the shell. Using eq $8 \mathrm{a}$ in ref 37 we can model our observed frequency shift with a surface melting layer starting from $6 \%$ at $T_{\text {trans }}$ and extending to $10 \%$ in an interval of $0.15 T_{\text {red }}$ before the damping due to sintering blurs the data. This is in the range of thickness as the prediction of the calculations ${ }^{6}$ or molecular dynamics studies. ${ }^{38}$

In conclusion we have found a phase transformation of supported gold particles at a very low temperature compared to the melting point of the particles. The transformation manifests itself as a discontinuous change of the vibrational parameters, in particular the phase and the frequency of the breathing mode of the particle. The vibrational damping increases at the transition and further at higher temperatures, where sintering occurs. These observations are in agreement with the development of a core-shell structure of the particle, 


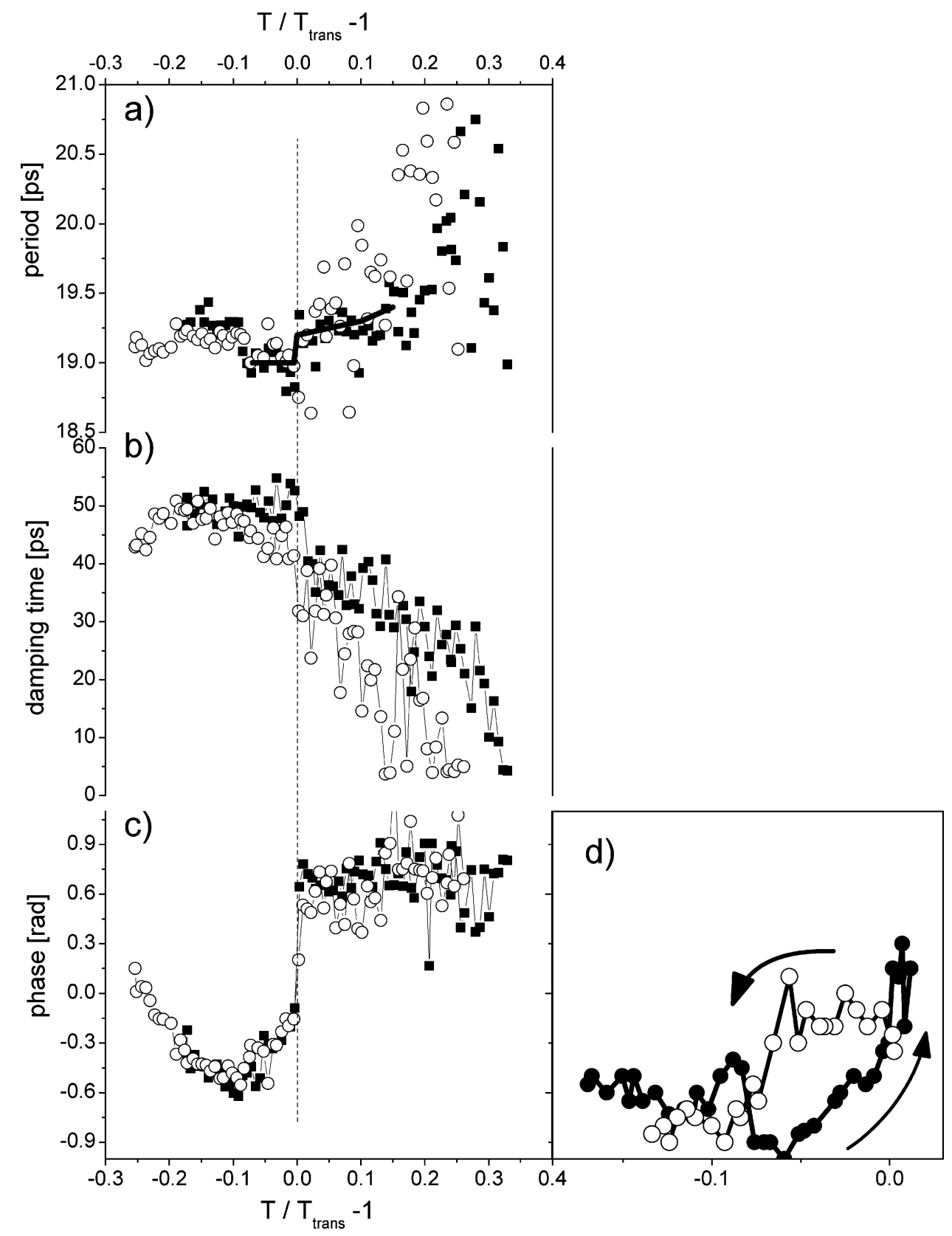

Figure 5. Parameters of the fits to the transient reflectivity by a damped cosine function for two different laser powers of $150 \mathrm{~mW}$ (solid symbols) and $320 \mathrm{~mW}$ (open symbols) as function of the reduced temperature $T / T_{\text {trans }}$ : (a) oscillation period; (b) damping time; (c) phase. The line in the upper figure shows the calculated change of the oscillation period for a thin surface melting layer. The trace in (d) is the phase during a temperature reversal from heating (solid symbols) to cooling (open symbols) shortly above the transition temperature.

with the surface melting layer acting a liquid viscous shell. Additionally the transition is found to be (partially) reversible. The low temperature of the transition is surprising, as surface melting for gold would only be expected at much higher temperatures. It can, on the other hand, explain recent observations of other groups, showing that shape transformation in gold particles such as nanorods can take place in the same temperature interval. ${ }^{8,9,39}$ A further point to be raised would be the time scale needed for the establishment of surface melting. A number of studies investigates the structural properties of particles upon high laser excitation. The energy dissipation occurs within a time range from hundreds of picoseconds to nanoseconds, ${ }^{13,40}$ which could be long enough to allow surface melting to occur. The appearance of surface melting for such pulsed excitation has not been seen yet. ${ }^{11,41,42}$ Finally a number of different technologically relevant processing steps for nanoparticle fabrication or function may involve such moderately elevated temperature, questioning stability issues.

Acknowledgment. We thank C. Janke for technical support and I. Vartanyants for discussions. This work was supported by a grant from the Ministry of Science, Research and the Arts of Baden-Württemberg, the Center for Junior Research Fellows of the University of Konstanz and the SFB 513, and by Gigaoptics GmbH. 


\section{References}

(1) Kreibig, U.; Vollmer, M Optical Properties of Metal Clusters; Springer: Berlin, 1995.

(2) Link, S.; El-Sayed, M. A. Int. Rev. Phys. Chem. 2000, 19, 409 and references therein.

(3) Burda, C.; Chen, X.; Narayanan, R.; El-Sayed, M. A. Chem. Rev. 2005, 105, 1025-1102.

(4) Rosi, N. L.; Mirkin, C. A. Chem. Rev. 2005, 105, 1547-1562.

(5) Buffat, P.; Borel, J. P. Phys. Rev. A 1976, 13, 2287.

(6) Sakai, H. Surf. Sci. 1996, 351, 285.

(7) Bachels, T.; Güntherodt, H.-J.; Schäfer, R. Phys. Rev. Lett. 2000, $85,1250$.

(8) Inasawa, S.; Sugiyama, M.; Yamaguchi, Y. J. Phys. Chem. B 2005, 109, 3103.

(9) Petrova, H.; Perez Juste, J.; Pastoriza-Santos, I.; Hartland, G. V.; Liz-Marzan, L. M.; Mulvaney, P. Phys. Chem. Chem. Phys. 2006, 8,814 .

(10) Link, S.; Wang, Z. L.; El-Sayed, M. A. J. Phys. Chem. B 2000, 104, 7867.

(11) Hartland, G. V.; Hu, M.; Sader, J. E. J. Phys. Chem. B 2003, 107, 7472.

(12) Hu, M.; Petrova, H.; Hartland, G. V. Chem. Phys. Lett. 2004, 391, 220.

(13) Plech, A.; Kotaidis, V.; Grésillon, S.; Dahmen, C.; von Plessen, G. Phys. Rev. B 2004, 70, 195423.

(14) Plech, A.; Kotaidis, V.; Lorenc, M.; Boneberg, J. Nat. Phys. 2006, 2, 44 .

(15) Kofman, R.; Cheyssac, P.; Aouaj, A.; Lereah, Y.; Deutscher, G.; Ben-David, T.; Penisson, J. M.; Bourret, A. Surf. Sci 1994, 303, 231

(16) Tartaglino, U.; Zykova-Timan, T.; Ercolessi, F.; Tosatti, F. Phys. Rep. 2005, 441, 291.

(17) Ercolessi, F.; Andreoni, W.; Tosatti, E. Phys. Rev. Lett. 1991, 66, 911.

(18) Wang, Z. L.; Petroski, J. M.; Green, T. C.; El-Sayed, M. A. J. Phys. Chem. B 1998, 102, 6163.

(19) Perner, M.; Bost, P.; Lemmer, U.; von Plessen, G.; Feldmann, J.; Becker, U.; Mennig, M.; Schmitt, M.; Schmidt, H. Phys. Rev. Lett. 1997, 78, 2192.

(20) Hodak, J. H.; Martini, I.; Hartland, G. V. Chem. Phys. Lett. 1998, 284, 135.

(21) Lamb, H. Proc. London Math. Soc. 1882, 13, 189.

(22) Tamura, A.; Higeta, K.; Ichinokawa, T. J. Phys. C 1982, 15, 4975.
(23) Voisin, C.; Del Fatti, N.; Christofilos, D.; Valleé, F. Appl. Surf. Sci. 2000, 164, 131.

(24) Hartland, G. V. J. Chem Phys. 2002, 116, 8048.

(25) Arbouet, A.; Voisin, C.; Christofilos, D.; Langot, P.; Del Fatti, N.; Valleé, F.; Lermé, J.; Celep, G.; Cottancin, E.; Gaudry, M.; Pellarin, M.; Broyer, M.; Maillard, M.; Pileni, M. P.; Treguer, M. Phys. Rev. Lett. 2003, 90, 177401.

(26) Elzinga, P. A.; Lytle, F. E.; Jian, Y.; King, G. B.; Laurendeau, N. M. Appl. Opt. 1987, 26, 4303.

(27) Bartels, A.; Hudert, F.; Janke, C.; Dekorsy, T.; Köhler, K. Appl. Phys. Lett. 2006, 88, 041117.

(28) Bartels, A.; Cerna, R.; Kistner, C.; Thoma, A.; Hudert, F.; Janke, C.; Dekorsy, T. Submitted for publication in Rev. Sci. Instrum.

(29) Plech, A.; Salditt, T. In Handbook of polyelectrolytes and their applications; Nalwa, H. S., Tripathy, S., Eds.; American Scientific Publishers: Valencia, CA, 2002; Vol. 1.

(30) Schmitt, J.; Mächtle, P.; Eck, D.; Möhwald, H.; Helm, C. A. Langmuir 1999, 15, 3256 and supporting information.

(31) Del Fatti, N.; Voisin, C.; Chevy, F.; Vallée, F.; Flytzanis, C. J. Chem. Phys. 1999, 110, 11484.

(32) Perner, M.; Grésillon, S.; März, J.; von Plessen, G.; Feldmann, J.; Porstendorfer, J.; Berg, K.-J.; Berg, G. Phys. Rev. Lett. 2000, 85, 792.

(33) Nie, S.; Wang, X.; Park, H.; Clinite, R.; Cao, J. Phys. Rev. Lett. 2006, 96, 025901.

(34) Hartland, G. V.; Hu, M.; Wilson, O.; Mulvaney, P.; Sader, J. E. J. Phys. Chem. B 2002, 106, 743.

(35) Kirakosyan, A. S.; Shahbatyan, T. V. Appl. Phys. B 2006, 84, 117.

(36) Hodak, J. H.; Henglein, A.; Hartland, G. V. J. Phys. Chem. B 2000, 104, 5043.

(37) Sader, J. E.; Hartland, G. V.; Mulvaney, P. J. Chem. Phys. B 2002 106, 1399.

(38) Ding, F.; Rosén, A.; Curtarolo, S.; Bolton, K. Appl. Phys. Lett. 2006, $88,133110$.

(39) Kana, C.; Wang, G.; Zhu, X.; Li, C.; Cao, B. Appl. Phys. Lett. 2006, 88, 071904.

(40) Plech, A.; Grésillon, S.; von Plessen, G.; Scheidt, K.; Naylor, G. Chem. Phys. 2004, 299, 183.

(41) Hartland, G. V. Annu. Rev. Phys. Chem. 2006, 57, 403.

(42) Kotaidis, V.; Dahmen, C.; von Plessen, G.; Springer, F.; Plech, A. J. Chem. Phys. 2006, 124, 184702.

NL070187T 